\title{
Questioning the aloe vera plant and apple memristors
} \author{
Edmund Pedersen Wood ${ }^{1,2}$, Daniel Åsen ${ }^{1,2}$ \\ 1. Department of Physics, University of Oslo, Oslo, Norway \\ 2. These authors contributed equally to the work \\ 3. E-mail any correspondence to: oliverpa@mail.uio.no
}

Oliver Pabst ${ }^{1,3}$, Steinar Andersen ${ }^{1,2}$, Soban Ali Bhatti ${ }^{1,2}$, Jørgen Brevik ${ }^{1,2}$, Simen Anthony Fallaas ${ }^{1,2}$, Mads Fjeldstad ${ }^{1,2}$, Artiom Gubaidulin ${ }^{1,2}$, Kjetil Vermundsen Madsen ${ }^{1,2}$, Mats Ricardo Nomedal ${ }^{1,2}$, Sondre Fortun Slettemoen ${ }^{1,2}$, Halvard Yri Adriaenssens ${ }^{1,2}$, Sean Andre Hansen ${ }^{1,2}$, Tommy Myrvik ${ }^{1,2}$, Eivind Rostad ${ }^{1,2}$, Torleif Skår ${ }^{1,2}$, Kristian Tuv ${ }^{1,2}$, Sebastian

\begin{abstract}
Non-linear electrical properties of a (biological) tissue can be revealed by non-linear electrical measurements, which means that the applied stimulus itself affects the measurement. If resulting voltage-current plots exhibit pinched hysteresis loops, the underlying tissue may be classified as a memristor, a state dependent resistor. The aloe vera plant and apples have been found to be memristors. However, polarization processes on the electrodes are also non-linear and may affect the measurement. Apples and aloe vera conduct electrical current very well and it is likely that the recordings are actually dominated by the polarization impedance of the electrodes. Here, we study the non-linear properties of aloe vera and apples with two different measurement electrode types. Furthermore, we measured also on the extracted liquids from one aloe vera leaf and one apple, leading to similar results. We concluded, unlike previous studies on these subjects, that the memristive properties originate from electrochemical reactions on the electrodes rather than the apples or aloe vera themselves.
\end{abstract}

Keywords: Bioimpedance; non-linear electrical properties; memristor; electrodes; cyclic voltammetry

\section{Introduction}

The term memristor ("memory resistor") was envisioned by Leon Chua [1] in 1971 and first developed by HP Labs in 2008 [2]. The memristance, $M$, (in analogy to resistance) of a memristor is dependent on one or more internal state variables. The voltage, $v$, and the current, $i$, are related via the state dependent ohm's law

$$
v=M(\boldsymbol{x}) i
$$

with $\boldsymbol{x}$ as the vector of the internal state variables. The change of $\boldsymbol{x}$ is described by the state equation

$$
d \boldsymbol{x} / d t=f(\boldsymbol{x}, i) \cdot i .
$$

A memristor has to exhibit pinched hysteresis loops in the V-I plot with the pinched point at the origin of the coordinates [3]. Furthermore, the lobe area of the loop decreases with increased frequency and if the frequency goes towards infinity, the pinched hysteresis loop should shrink to a single valued function [4].

Volkov et al. demonstrated memristive properties in apples [5] and in the aloe vera plant [6]. Here, we study the memristive properties of the same objects by using our methods (three electrode system that enables monopolar recordings and silver/silver chloride $(\mathrm{Ag} / \mathrm{AgCl})$ electrodes). We also used transimpedance amplifiers for the reading of the current instead of single resistors in series. Furthermore, we recorded simultaneously with a stainless steel needle electrode to compare also with the results of Volkov et al. who used coated platinum needle electrodes. We found that dependent on which electrode type was used, there are large differences in the results and two possible explanations 
arise. If the recorded non-linear electrical properties are dominated by the apple or aloe vera, the differences between both electrodes may originate from the different current densities around each electrode. The current density around the needle electrode is much higher and may cause stronger non-linear effects. However, another possibility is that electrochemical reactions on the electrodes themselves are the origin of the non-linear properties. In this case it is to question whether there are memristive properties in the apples and the aloe vera plant itself. We conducted therefore a second experiment in which we placed the electrodes not in the apples and the aloe vera plant, but only in the liquids that were obtained from both. We found non-linear electrical properties that were similar to the experiments on the whole apple and the aloe vera plant. Finally, we did measurements in apple juice from concentrate and obtained similar results.

\section{Materials and methods}

\section{Samples}

We did measurements on three different aloe vera plants (family "Asphodelaceae", on two different leaves each) and on four different apples (type Torstein) in October and November 2018 at the University of Oslo. Each apple measurement was first done on the entire piece followed by recording on a smaller piece from the same apple (about 1 $\mathrm{cm}$ thickness) that was cut off (see Fig. 1c). The measurements on the apple liquid and aloe vera liquid were conducted once each in May 2019. The apple liquid was obtained from a peeled apple (sort "evelina") that was crushed in a blender (the core was removed) and the resulting apple mush was placed in a coffee filter. Manual squeezing enabled obtaining some apple liquid through the filter. The content of one aloe vera leaf (family "Asphodelaceae") was picked with a spoon. Filtration of the pure liquid (without any solid substance) by using a coffee filter did not work that well. In the end we excluded any chunk manually with a spoon. Both liquids were filtrated into the truncated bottom of a plastic cup (diameter of around 5 $\mathrm{cm}$, see Fig. 1c). The measurements on the apple juice (100 $\%$ juice from concentrate, company Sunniva) were done once in July 2019.

\section{Electrodes}

Dry sintered silver/silver chloride electrodes (from Wuhan Greentek PTY LTD) that had a cylindrical shape and were not prewired were used as M1, ref and CC electrode. Each wire was manually connected via a shrinking tube. The diameter of each electrode was $2 \mathrm{~mm}$ and the length was about 2 to 3 $\mathrm{mm}$ (determined by the position of the isolation of the shrinking tube that covered the upper part of the electrode). The non-isolated tip of the medical stainless needle electrode had a diameter of around $0.2 \mathrm{~mm}$ and a length of around $0.8 \mathrm{~mm}$. All electrodes were cleaned with ethanol before use.

The electrodes for the aloe vera measurements were placed central at the upper side of the chosen leaf (see Fig. 1c). The electrodes $M 1, M 2, C C$ and ref were placed at about $5 \mathrm{~cm}, 6 \mathrm{~cm}, 8 \mathrm{~cm}$ and $9 \mathrm{~cm}$ from the trunk, respectively.

On the full apple, the electrodes where placed about halfway up. In relation to the measurement electrode M1 along the circumference, the reference electrode, the CC electrode and the $\mathrm{M} 2$ electrode were placed about $2 \mathrm{~cm}, 3$ $\mathrm{cm}$ and $6 \mathrm{~cm}$ apart, respectively (see left photograph of Fig. 1c). For the apple piece measurements, the M1 electrode was placed at the left outside (see middle photograph of Fig. 1c), and the electrodes ref, $\mathrm{CC}$, and $\mathrm{M} 2$ were placed about $1.5 \mathrm{~cm}, 2.5 \mathrm{~cm}$ and $5 \mathrm{~cm}$ apart. Within all liquid measurements, the CC and the ref electrodes were placed on one side of the cup and both measurement electrodes were placed on the opposite site of the cup with around $1 \mathrm{~cm}$ distance to each other.

\section{Instrumentation}

The same custom-built measurement system as used in [7] was used here (see Fig. 1b), based on a data acquisition card (DAQ, type USB-6356 from National instruments, controlled by custom made software written in NI LabVIEW version 2014). The transimpedance amplifiers in the reading channels convert the current into a voltage that can be read by the DAQ. The feedback resistors $R_{\mathrm{fb} 1}$ and $R_{\mathrm{fb} 2}$ determine the gain and have both a value of $56 \mathrm{k} \Omega$ for the measurements on the aloe vera leaf. Much higher currents were expected for the measurements on the apples (known from pilot studies) and a resistor with a resistance value of $0.56 \mathrm{k} \Omega$ was chosen as $R_{\mathrm{fb} 1}$ instead. For the measurements on the apple juice and aloe vera liquid the feedback resistances $R_{\mathrm{fb} 1}=0.56 \mathrm{k} \Omega$ and $R_{\mathrm{fb} 2}=5.6 \mathrm{k} \Omega$ were used. The capacitance $\mathrm{C}_{\mathrm{fb}}=4.7 \mathrm{nF}$ in addition to each transimpedance amplifier enables reduction of high frequency noise. Signal generation and reading were performed with 2000 samples per period.

\section{Experimental procedure}

Different alternating current $(A C)$ voltage waveforms (sinusoidal with different amplitudes and triangular, see Fig. 1a) were applied, and a frequency sweep $(0.005 \mathrm{~Hz}, 0.05 \mathrm{~Hz}$ and $0.5 \mathrm{~Hz}$ ) was done for each. Each recording was done for two periods.

The sweep over the voltage waveform and the sweep over the frequencies were performed automated in randomized order for the measurements on the apples and aloe vera plants, and in manually chosen order for the measurements in the liquids. 
a) Voltage stimuli

\begin{tabular}{|l|l|c|c|}
\hline \multicolumn{1}{|c|}{ object } & waveform & Amplitudes [V] & $\mathrm{f}[\mathrm{Hz}]$ \\
\hline $\begin{array}{l}\text { Aloe Vera } \\
\text { leaf }\end{array}$ & sinusoidal & $2.5,5$ & \\
& triangular & 5 & \\
\hline $\begin{array}{l}\text { Apple, } \\
\text { apple } \\
\text { piece }\end{array}$ & sinusoidal & $\begin{array}{c}\text { 2 (full apple only), 4, } \\
\text { 6 (apple piece only) }\end{array}$ & 0.005, \\
\hline $\begin{array}{l}\text { Aloe Vera } \\
\text { juice }\end{array}$ & $\begin{array}{l}\text { triangular } \\
\text { sinusoidal }\end{array}$ & 4 & 0.05, \\
\hline $\begin{array}{l}\text { triangular } \\
\text { Apple }\end{array}$ & sinusoidal & $1,2,2.5,4,4$ & 0.5 \\
\hline juice & triangular & 2 & \\
\hline
\end{tabular}

b) Instrumentation

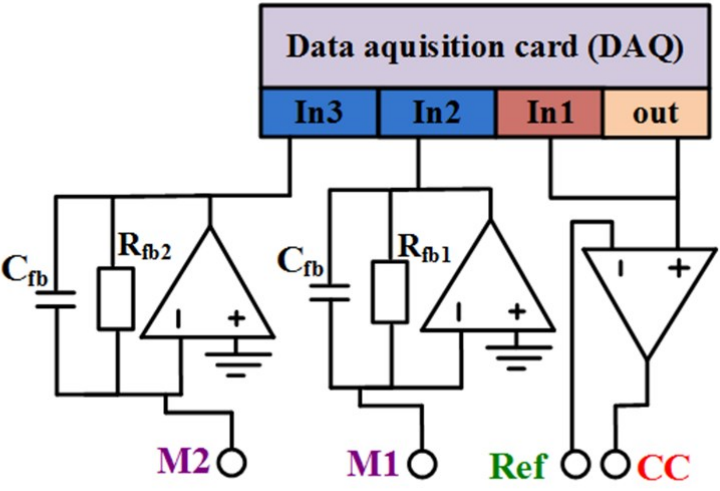

c) Electrode placement
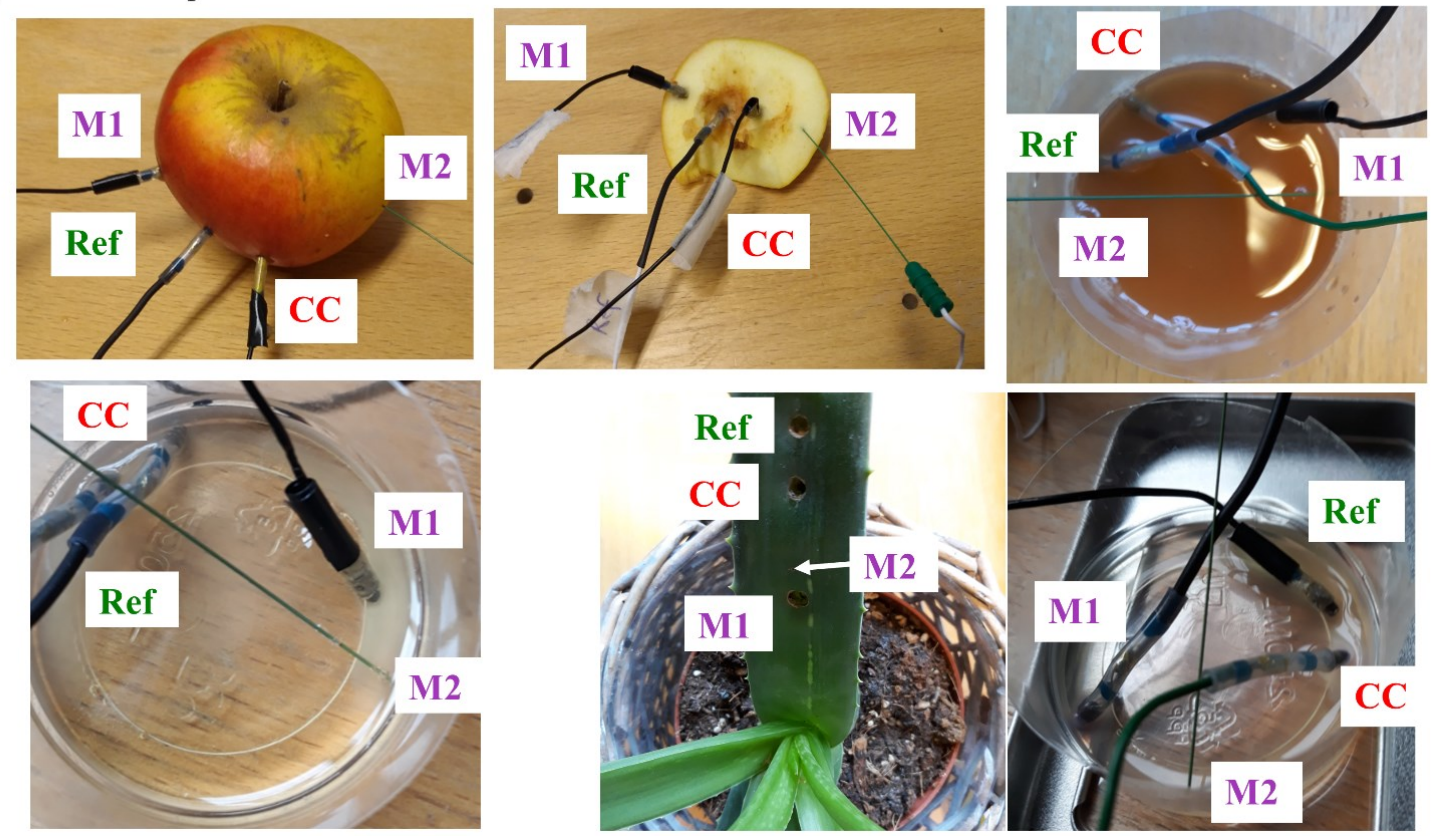

Fig 1: Methods, a) Voltage stimuli that were used in the experiment. The voltages used on the apple juice (manually obtained from one apple) are listed in the table. The measurements on the apple juice from concentrate were only done with sinusoidal voltages with amplitudes of $2.5 \mathrm{~V}$ and $4 \mathrm{~V}$. b) Schematic of the measurement instrumentation. The recordings under the measurement electrodes $\mathrm{M} 1$, and $\mathrm{M} 2$ are monopolar and were done simultaneously, which was enabled by a three electrode setup (see [8]) with "CC" as the current carrying electrode and "Ref" as the reference electrode. c) Electrode placement on the whole apple (left), on the apple piece (middle), the apple liquid (right), apple juice from concentrate (bottom left), aloe vera leave (bottom middle) and the aloe vera liquid (bottom right).

\section{Results}

The recordings from the aloe vera plants and the extracted liquid (see Figs. 2 and S1) and from the apples and apple juice (see Figs. 3 and S2) are non-linear and showed pinched hysteresis loops in the voltage current presentation. The recordings obtained with the stainless steel needle are more non-linear than for the silver/silver chloride electrodes.

Pinched hysteresis loops with pinched point in the third quadrant or two pinched points can be observed from the recordings with the stainless steel electrode. Pinched hysteresis loops with pinched point in the first quadrant can be observed from the recordings with the silver/silver chloride electrode. The obtained currents from the aloe vera extract were higher than the currents obtained from the aloe vera leaf.

However, the shape of the pinched hysteresis loops obtained from both subjects are quite similar. The same observation can be done between the apple juice and the whole apple. In addition, the measurements on the apple or apple juice in comparison with the aloe vera or aloe vera extract obtained from the stainless steel needle electrode, look quite similar.

With increasing frequency, all recordings with the silver/silver chloride electrode (see Figs. S1 and S2) tend to be single valued functions in the voltage current plane while the recordings with the stainless steel electrode are still nonlinear even at a frequency of $0.5 \mathrm{~Hz}$.

A white layer was observed on the silver/silver chloride electrodes ( $M$ and $C C$ ) after the measurements in apple juice. Additional measurements in diluted $\mathrm{HCL}$ acid and in apple juice from concentrate with a platinum wire were done (see Fig. S3b) and also showed pinched hysteresis loops. 
$\mathrm{Ag} / \mathrm{AgCl}$ electrode
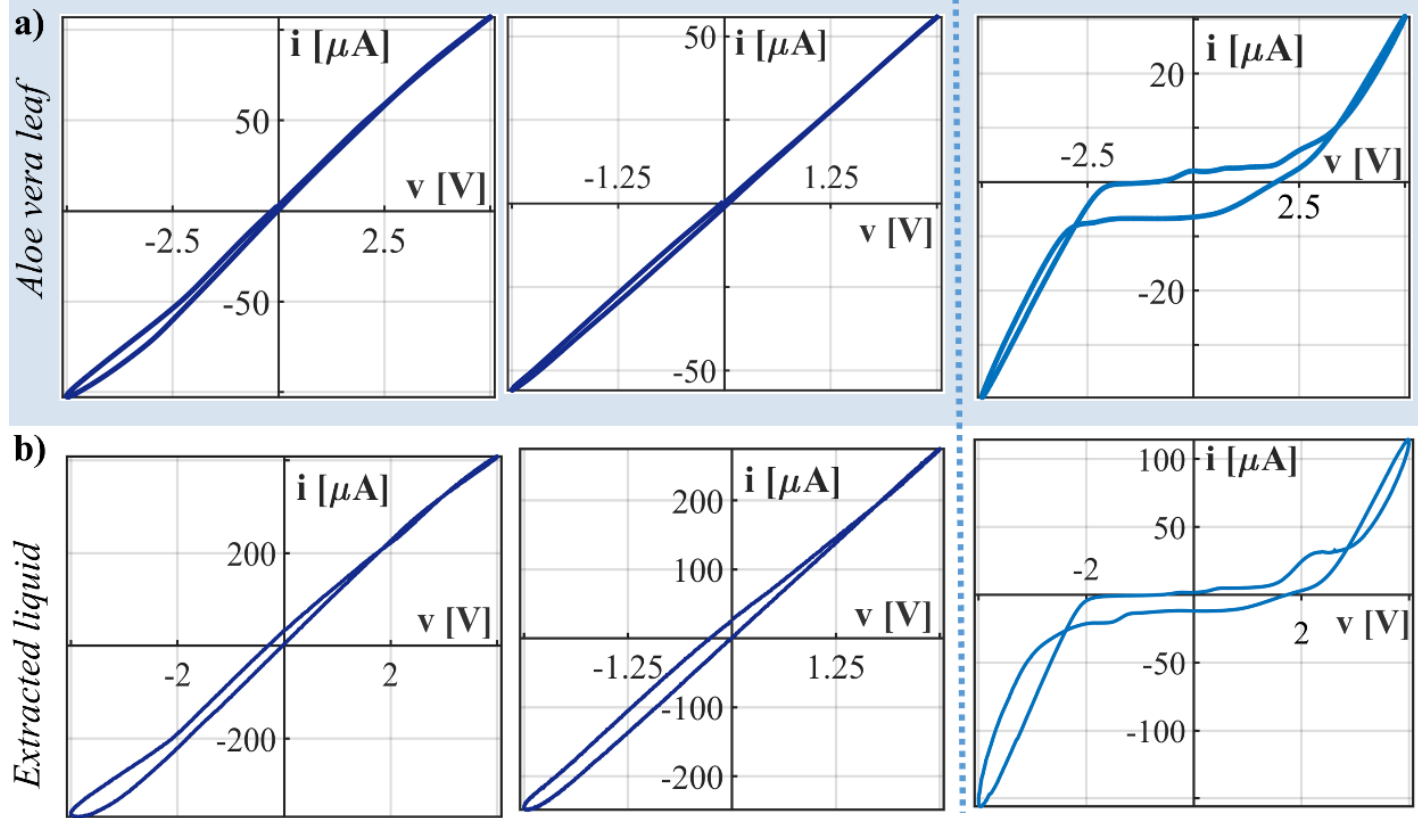

Stainless steel needle electrode
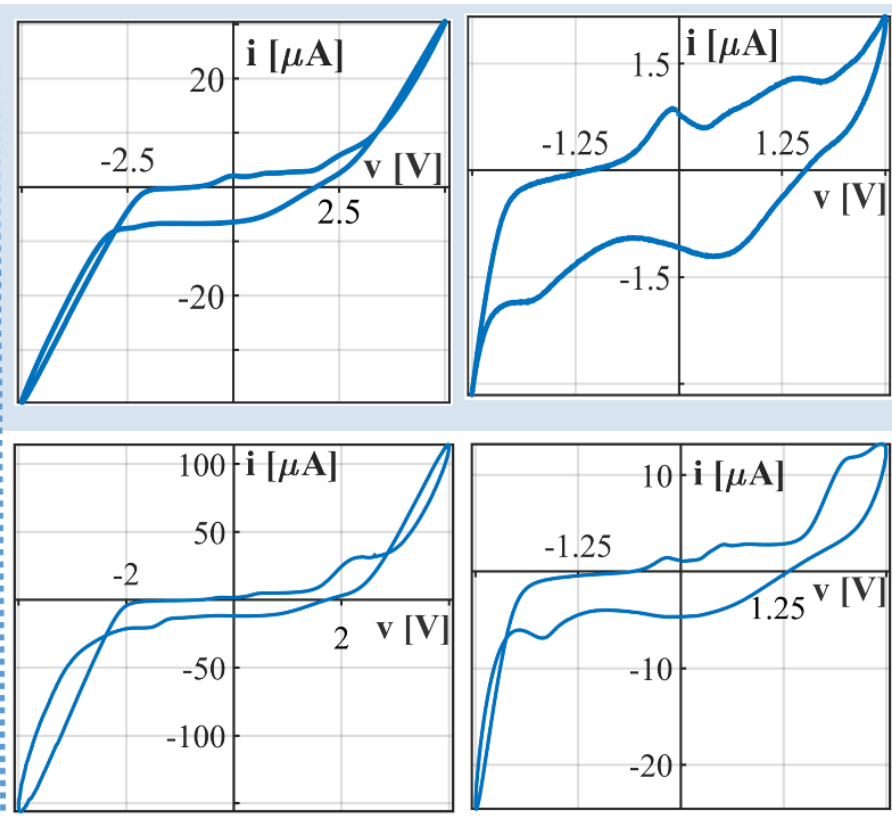

Fig. 2: Voltage current plots recorded from the aloe vera plants, always shown for the second period of each measurement. The dark blue plots represent the results from recordings with the silver/silver chloride electrode and the light blue plots from the recordings with the medical stainless steel needle electrode. a) Results from one leaf of one aloe vera plants (A) shown for applied sinusoidal voltages with amplitudes of $5 \mathrm{~V}$ and $2.5 \mathrm{~V}$ and frequency equal to $0.005 \mathrm{~Hz}$. The recordings obtained by the silver/silver chloride electrode are slightly non-linear. Pinched hysteresis loops with small loop area and pinched point position in the first quadrant were obtained. b) Results from the liquid of one leaf of one aloe vera plant shown for applied sinusoidal voltages with amplitudes of $4 \mathrm{~V}$ and $2.5 \mathrm{~V}$ and a frequency of $0.005 \mathrm{~Hz}$.

\section{Discussion}

One could argue that the measurements on the apple and the aloe vera reveal underlying memristors since pinched hysteresis loops can be shown for different amplitudes and signal shapes (see Figs. 2, 3, S1, and S2). Shifts of the pinched point away from the origin of coordinates or two pinched points can be explained by a capacitor in parallel with the underlying memristors [9].

However, assuming that the results represent a memristor, the question is what the actual mechanism behind these non-linear properties is. Volkov et al. explained the memristive properties they found in apples and aloe vera by voltage gated potassium-channels that are activated as soon as the voltage is high enough $[6,10]$. By our measurements, we cannot exclude that this is happening. However, if these processes are happening, these are not the dominating mechanism since similar results can be achieved between the apple and apple juice and between the aloe vera leaf and the extract from the aloe leaf. Furthermore, in the apple juice from concentrate or the hydrochloric acid (see additional measurements in Fig. S3), there are no living cells and the memristive properties cannot be explained by voltage gated potassium channels.

The conductances of the apples, the apple juice, the aloe vera, and extract of the aloe vera leaf are quite high and there is a strong indication that the electrode impedance of the electrodes actually dominates the measurements. The origin of the memristive properties is likely to be electrochemical reactions on the electrodes themselves as in cyclic voltammetry [11]. The results between the silver/silver chloride electrode and the stainless steel needle electrode are very different from each other which can be explained by different reactions. At the stainless steel needle electrode and also the platinum electrode (additional measurement, see Fig. S3) a redox reaction of water is happening with

$$
\begin{aligned}
& 2 \mathrm{H}_{2} \mathrm{O} \rightarrow \mathrm{O}_{2}+4 \mathrm{H}^{+}+4 \mathrm{e}^{-} \quad \text { (Oxidation) } \\
& 2 \mathrm{H}^{+}+2 \mathrm{e}^{-} \rightarrow \mathrm{H}_{2} \quad \text { (Reduction) }
\end{aligned}
$$

while it is likely that the equilibrium between silver and silver chloride is shifted with

$$
\mathrm{e}^{-}+\mathrm{AgCl} \rightarrow \mathrm{Ag}+\mathrm{Cl}^{-}
$$

at the corresponding electrodes.

Anyway, in a bipolar measurement (with two electrodes), the polarization impedances of both electrodes are contributing and the overall setup can be modelled by a serial connection of two memristors that are in opposite direction to each other. With the use a three-electrode system, that allows monopolar recordings under the measurement electrode, the polarization impedance of only one electrode can be revealed.

To find out whether there are actually memristive properties in the apple, the apple juice, the aloe vera leaf, and the aloe vera juice themselves, a four electrode system (two current carrying electrodes and two potential pickup electrodes) would be useful. 
$\mathrm{Ag} / \mathrm{AgCl}$ electrode
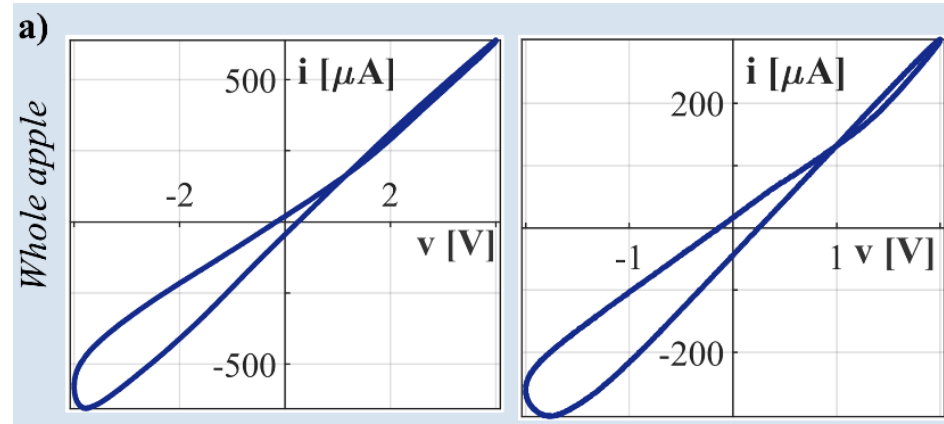

b)
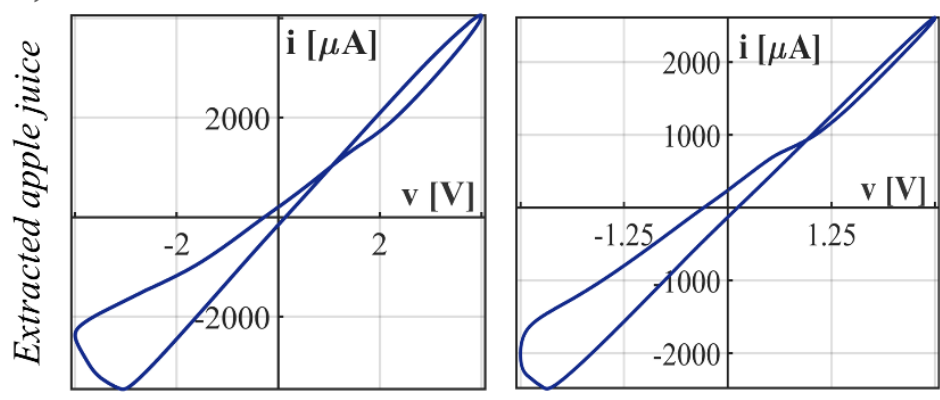

c)
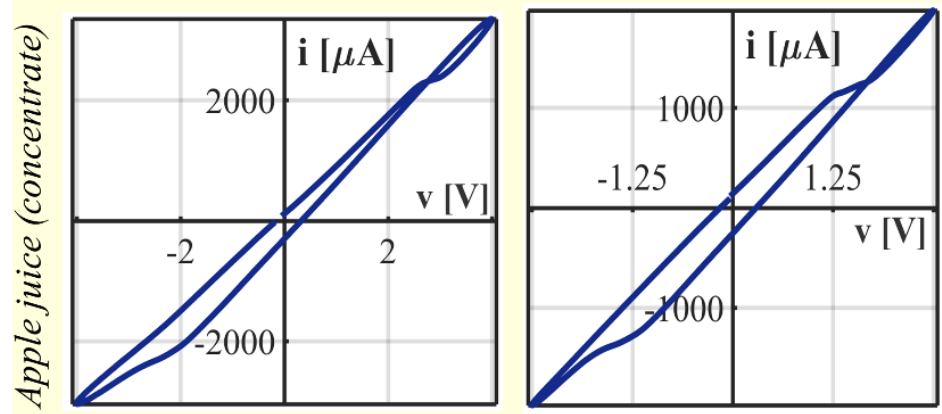

Stainless steel needle electrode
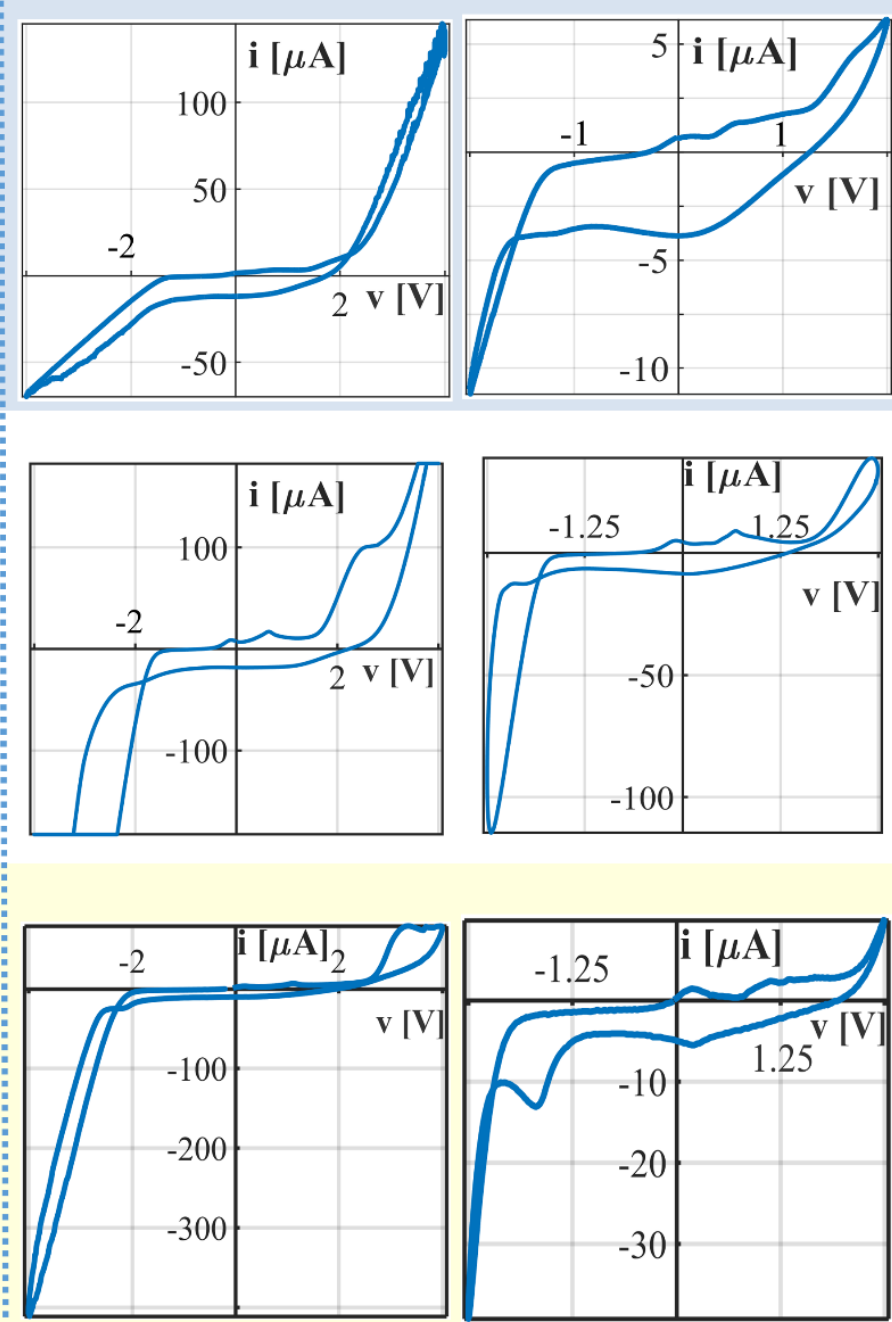

Fig. 3: Voltage current plots recorded from the apples, always shown for the second period of each measurement. The dark blue plots represent the results from recordings with the silver/silver chloride electrode and the light blue plots from the recordings with the stainless steel needle electrode. All shown results were obtained by applying sinusoidal voltages with a frequency of $0.005 \mathrm{~Hz}$ and different amplitudes. a) Results from the measurements on one whole apple (A) (used amplitudes were $4 \mathrm{~V}$ and $2 \mathrm{~V}$ ). Similar results were obtained from two other apples (B and C). The recordings from the apple D (see Fig. S2) were slightly more linear. b) Recordings from the apple juice that was manually extracted (used amplitudes were $4 \mathrm{~V}$ and $2.5 \mathrm{~V}$ ). The cutoff in the plot of the recording with the stainless steel needle at $4 \mathrm{~V}$ amplitude is due to saturation of the transimpedance amplifier. c) Recordings from the apple juice from concentrate (used amplitudes were $4 \mathrm{~V}$ and $2.5 \mathrm{~V})$.

In such a setup, no current flows through the pickup electrodes and one can be sure that the measured electrical properties are not due to electrode polarization.

\section{Conflict of interest}

Authors state no conflict of interest.

Data and materials availability

The recorded data have been deposited with figshare. These data can be obtained free of charge from:

https://figshare.com/s/725fedd043d15fed3b0b .

\section{Acknowledgement}

O.P. thanks Prof. Ola Nilsen for his advices on the electrochemistry and revising the manuscript.

\section{References}

1. Chua LO. Memristor-the missing circuit element. IEEE Transactions on circuit theory. 1971;18(5):507-19. https://doi.org/10.1109/tct.1971.1083337

2. Strukov DB, Snider GS, Stewart DR, Williams RS. The missing memristor found. nature. 2008;453(7191):80. https://doi.org/10.1038/nature06932

3. Chua LO. If it's pinched it's a memristor. Semiconductor Science and Technology. 2014;29(10):104001. https://doi.org/10.1088/0268-1242/29/10/104001

4. Adhikari SP, Sah MP, Kim H, Chua LO. Three fingerprints of memristor. IEEE Transactions on Circuits and Systems I: Regular Papers. 2013;60(11):3008-21. https://doi.org/10.1109/tcsi.2013.2256171

5. Volkov AG, Markin VS. Electrochemistry of Gala apples: Memristors in vivo. Russian Journal of Electrochemistry. 2017;53(9):1011-8. https://doi.org/10.1134/s1023193517090166 
6. Volkov AG, Reedus J, Mitchell CM, Tucket C, Forde-Tuckett V, Volkova MI, et al. Memristors in the electrical network of Aloe vera L. Plant Signalling \& Behavior. 2014;9(7):e29056. https://doi.org/10.4161/psb.29056

7. Pabst $O$, Martinsen $\varnothing \mathrm{G}$, Chua LO. The non-linear electrical properties of human skin make it a generic memristor. Scientific Reports. 2018;8(1):15806.

https://doi.org/10.1038/s41598-018-34059-6

8. Grimnes S. Impedance measurement of individual skin surface electrodes. Medical and Biological Engineering and Computing. 1983;21(6):750-5. https://doi.org/10.1007/bf02464038
9. Pabst $O$, Martinsen $\varnothing \mathrm{G}$. Simulation based comparison between a transversal and a tangential memristor model with a capacitance in parallel. PloS one. 2019;14(8). https://doi.org/10.1371/journal.pone.0221533

10. Volkov AG, Nyasani EK, Tuckett C, Blockmon AL, Reedus J, Volkova MI. Cyclic voltammetry of apple fruits: memristors in vivo. Bioelectrochemistry. 2016;112:9-15. https://doi.org/10.1016/j.bioelechem.2016.07.001

11. Elgrishi N, Rountree KJ, McCarthy BD, Rountree ES, Eisenhart TT, Dempsey JL. A practical beginner's guide to cyclic voltammetry. Journal of Chemical Education. 2017;95(2):197206. https://doi.org/10.1021/acs.jchemed.7b00361

\section{Supplementary information}
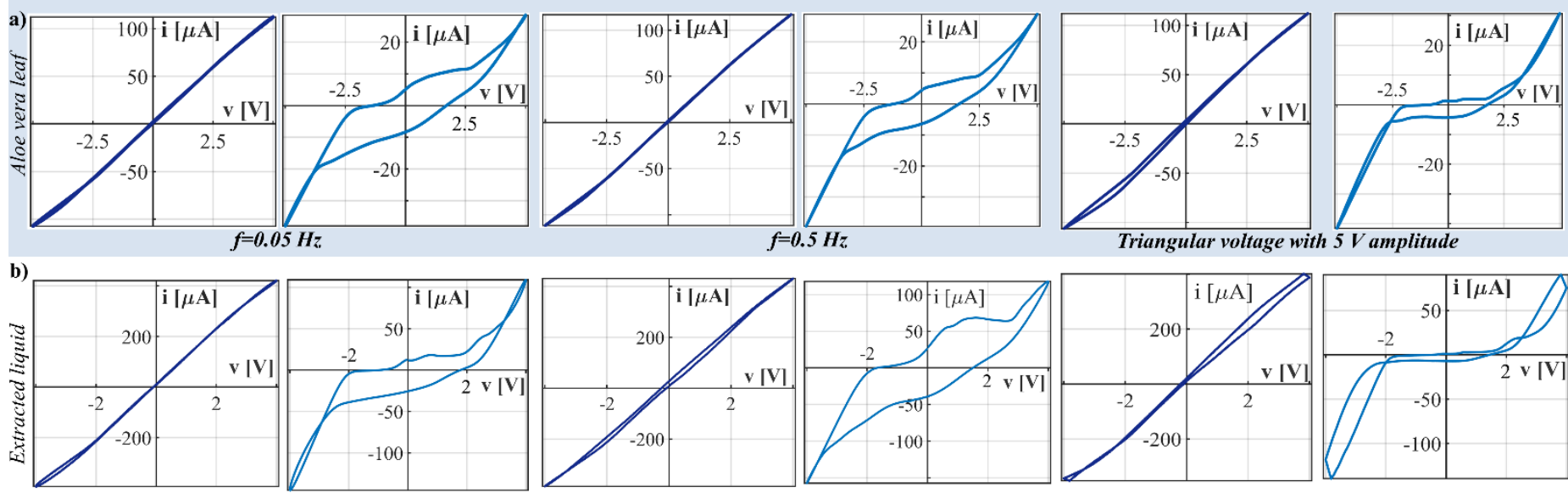

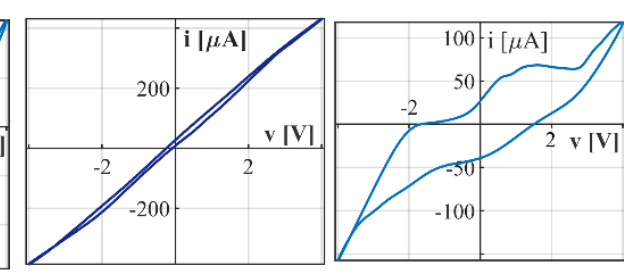

$f=0.5 \mathrm{~Hz}$

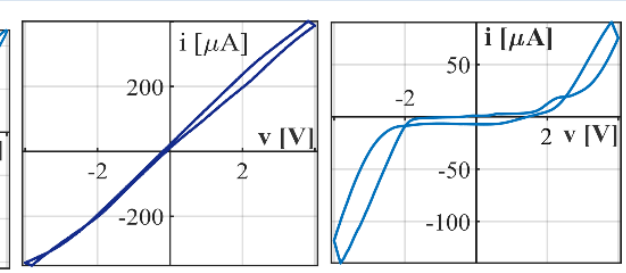

Triangular voltage with $4 \mathrm{~V}$ amplitude

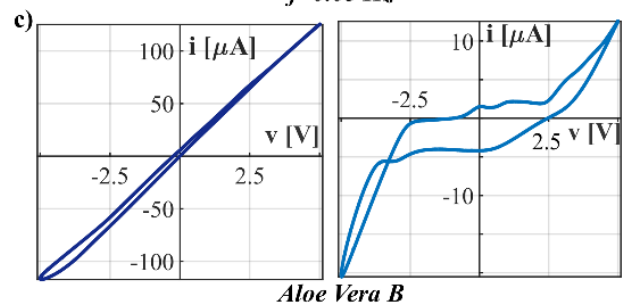

Fig. S1: Additional voltage current plots recorded from the aloe vera plants, always shown for the second period of each measurement. The dark blue plots represent the results from recordings with the silver/silver chloride electrode and the light blue plots from the recordings with the stainless steel needle electrode. a) Results from the leaf of one aloe vera plant (A) shown for applied sinusoidal voltage with an amplitude of $5 \mathrm{~V}$ and frequency equal to $0.05 \mathrm{~Hz}$ and frequency equal to $0.5 \mathrm{~Hz}$ and triangular voltage with $5 \mathrm{~V}$ amplitude and frequency of $0.005 \mathrm{~Hz}$. b) Results from the extracted liquid of one aloe vera leaf shown for applied sinusoidal voltage with an amplitude of $4 \mathrm{~V}$ and frequency of $0.05 \mathrm{~Hz}$ and $0.5 \mathrm{~Hz}$ and triangular voltage with an amplitude of $4 \mathrm{~V}$ and frequency of $0.005 \mathrm{~Hz}$. c) Results from the leaf of another aloe vera plant (B) shown for sinusoidal voltage with an amplitude of $5 \mathrm{~V}$ and frequency $\mathrm{f}=0.005 \mathrm{~Hz}$. 
a) apple juice

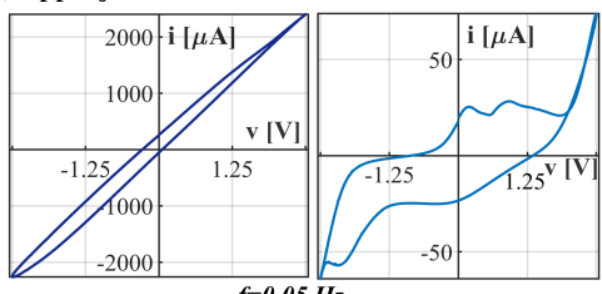

$f=0.05 \mathrm{~Hz}$

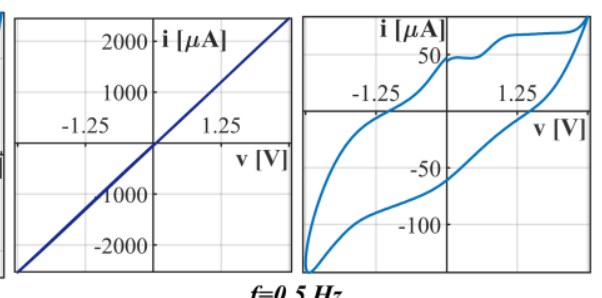

$f=0.5 \mathrm{~Hz}$

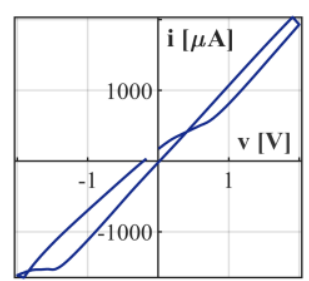

Triangular voltage with $2 \mathrm{~V}$ amplitude

b) Whole apple

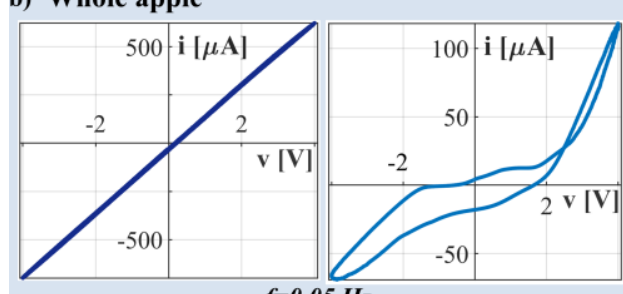

$f=0.05 \mathrm{~Hz}$

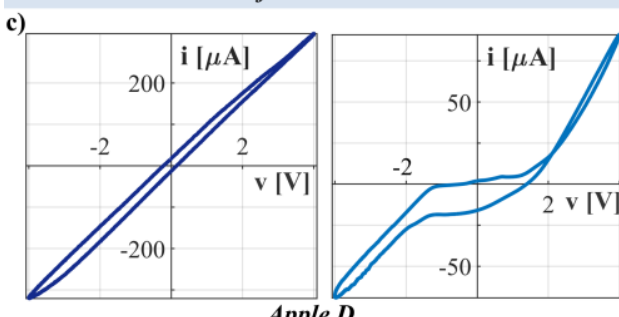

Apple D

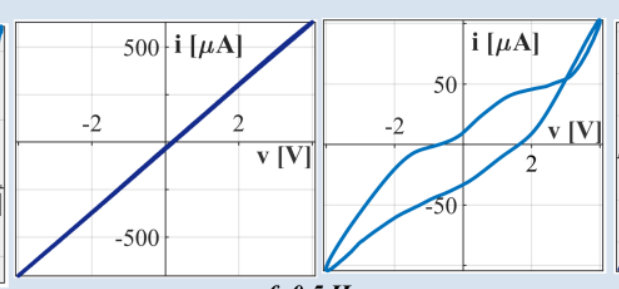

$f=0.5 \mathrm{~Hz}$

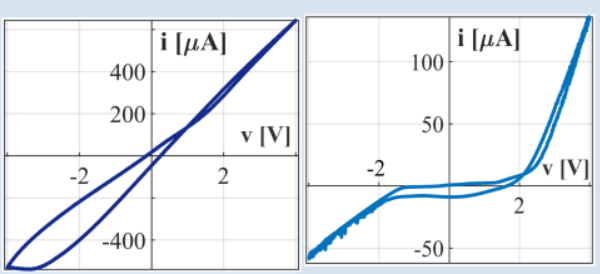

Triangular voltage with $4 V$ amplitude

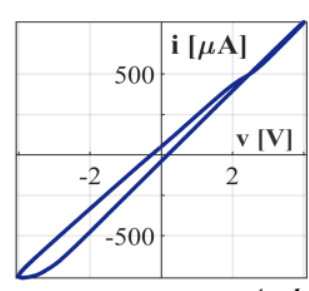

d)

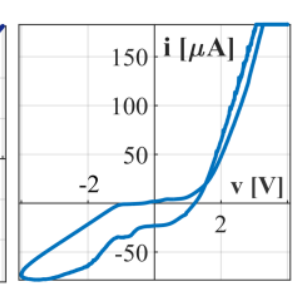

Apple piece

Fig. S2: Additional voltage current plots recorded from the apples, always shown for the second period of each measurement. The dark blue plots represent the results from recordings with the silver/silver chloride electrode and the light blue plots from the recordings with the stainless steel needle electrode. a) Results from the manual extracted apple juice shown for applied sinusoidal voltage with an amplitude of $2.5 \mathrm{~V}$ and frequency equal to $0.05 \mathrm{~Hz}$ and frequency equal to 0.5 $\mathrm{Hz}$ and triangular voltage with $2 \mathrm{~V}$ amplitude and frequency of $0.005 \mathrm{~Hz}$. b) Results from one whole apple (A) shown for applied sinusoidal voltage with an amplitude of $2.5 \mathrm{~V}$ and frequency of $0.05 \mathrm{~Hz}$ and $0.5 \mathrm{~Hz}$ and triangular voltage with an amplitude of $4 \mathrm{~V}$ and frequency of $0.005 \mathrm{~Hz}$. At frequencies of $0.05 \mathrm{~Hz}$ and $0.5 \mathrm{~Hz}$, the results from the silver/silver chloride electrode recordings are already linear, since a straight line in the voltage current plots can be observed. The needle electrode recordings are still non-linear at these frequencies. c) Results from another whole apple (D) and from a piece of an apple shown for sinusoidal voltage with an amplitude of $4 \mathrm{~V}$ shown for frequency $\mathrm{f}=0.005 \mathrm{~Hz}$. The recordings on the apple pieces are quite similar to the recordings at the whole apples except that the recorded currents are higher. Saturation of the operational amplifier in the measurement channel of the needle electrode is reached and currents larger than about $175 \mu$ A could not be read.

a) Diluted HCL acid

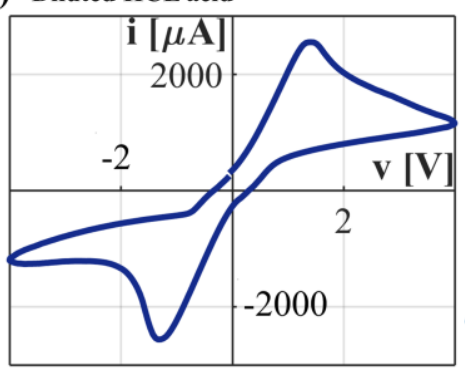

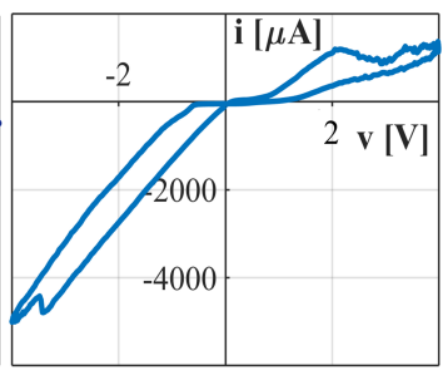

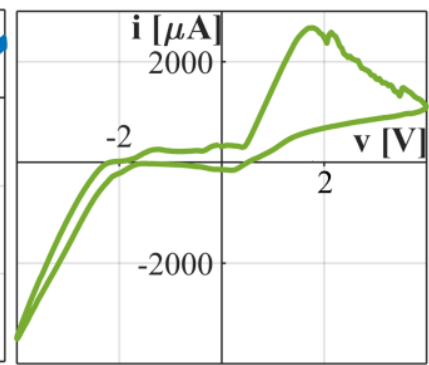

b) Platinum electrode in apple juice

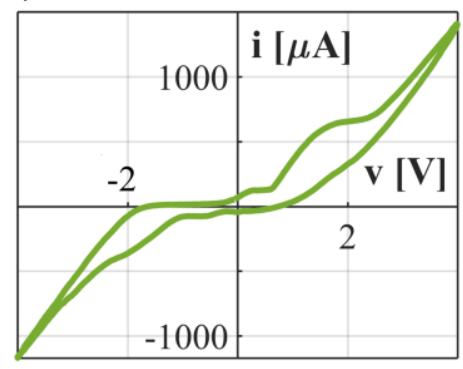

Fig. S3: Additional voltage current plots, always shown for the second period of each measurement. These extra measurements were done in December 2019 with a sinusoidal voltage with an amplitude of $4 \mathrm{~V}$ and a frequency of $0.005 \mathrm{~Hz}$. Results from the silver/silver chloride electrode, the stainless steel electrode and a platinum wire (diameter of $0.4 \mathrm{~mm}$ and length of $1 \mathrm{~cm}$ ) are presented as dark blue, light blue and green plots, respectively. Parts of the isolation of the stainless steel electrode were scratched which means that the active electrode area was larger than the electrode tip. The measurements were done after each other using only one reading channel with $\mathrm{Rfb}_{1}=0.56 \mathrm{k} \Omega$. a) Measurements in $\mathrm{HCL}$ acid that was diluted with deionized water. The overall $\mathrm{pH}$-value of the solution was 3 . Redox reaction of water is happening on all three electrode types. $\mathbf{b}$ ) Measurement with the platinum wire in apple juice from concentrate. 